\title{
Responses of woody plant Dalbergia odorifera treated with glycine betaine to drought and cold stresses: involvement of the alternative oxidase
}

\author{
E.H.M. CISSE (D), D.-D. LI, J. ZHANG, L.-Y. GUO, L.-F. MIAO*, and F. YANG* (D) \\ School of Ecological and Environmental Sciences, Hainan University; Center for Eco-Environmental Restoration \\ Engineering of Hainan Province; Key Laboratory of Agro-Forestry Environmental Processes and Ecological \\ Regulation of Hainan Province, Haikou, Hainan, 570228, P.R. China
}

*Corresponding authors: E-mails: mlf-sx@163.com, fanyangmlf6303@163.com

\begin{abstract}
Woody plants faced multiple abiotic stresses in forest plantation that can influence their growth and development. Dalbergia odorifera $\mathrm{T}$. Chen is a vulnerably endangered tree species, and references about its responses to abiotic stresses are very rare in literature. Furthermore, the mechanisms underlying the abiotic stress tolerance in plants induced by exogenous glycine betaine (GB) remains unclear. Indeed, the alternative oxidase (AOX) is one of the major components of antioxidant enzymatic machinery, and there are no studies that focused on the effects of GB on the amount of AOX protein in plants under drought or cold stresses. Thus, the aim of this study was to investigate the effect of exogenous GB on the phenotype, osmoprotectants, photosynthetic pigments, and antioxidant systems in D. odorifera under cold and drought stresses. The layout of the trial was a factorial experiment in a completely randomized design using two factors including abiotic stress (drought and cold) and GB. Moreover, the principal component analysis based on the measured parameters manifested how the selected ROS scavengers were dispersed throughout the treatments. The results showed huge beneficial impacts of GB on the phenotypic traits of $D$. odorifera; GB also influences positively the antioxidant machinery, photosynthetic pigments, redox-homeostasis, and water status in $D$. odorifera seedlings under both stresses. Moreover, exogenous GB affects more the AOX pathway in D. odorifera under cold stress than under drought stress.
\end{abstract}

Keywords: AOX, APX, CAT, chilling, chlorophyll, Dalbergia odorifera, drought, glycine betaine, homeostasis, oxidative stress, peroxidase, ROS, SOD.

\section{Introduction}

With global climate change, the research about the response of a plant to abiotic stresses is one of the most active themes in plant science. Indeed, climate change appears to have increased in recent years, and more changes are expected in the next decades concerning plant growth and adaptation to their environments (Cernoch and Kopecky 2020). Abiotic stresses such as drought or cold stress affect plants by harming the metabolic machinery; it can also perturb the equilibrium of the production and scavenging of reactive oxygen species (ROS) and malondialdehyde (MDA) in the plant cell, which alters negatively plant growth and development (Sharma et al. 2019). Stress tolerance in plants is correlated with different physiological changes including the accumulation of osmoprotectants and the increase of antioxidant activities (Yancey et al. 1994). Some of these adjustments are needed for enhancing the drought or cold tolerance in plants, comprise changes in genes expression and in membrane

$\overline{\text { Received }} 12$ March 2021, last revision 28 September 2021, accepted 22 October 2021.

Abbreviations: APX - ascorbate peroxidase; AsA - ascorbic acid; Car - carotenoid; CAT - catalase; Chl $a$ - chlorophyll $a$; Chl $b$ chlorophyll $b$; ci - intercellular $\mathrm{CO}_{2}$ concentration; GB - glycine betaine; GPX - glutathione peroxidase; MDA - malondialdehyde; POD - peroxidase; RC - relative conductivity; ROS - reactive oxygen species; RWC - relative water content; SOD - superoxide dismutase; WP - water potential (measured by dew-point method).

Acknowledgements: The present work was supported by the Hainan Provincial Natural Science Foundation of China (Nos. 320 RC507 and 317052), and the National Natural Science Foundation of China (Nos. 32060240 and 31660165 ).

Conflict of interest: The authors declare that they have no conflict of interest. 
composition and behaviour (Wanner and Junttila 1999, Sharma et al. 2019, Kumar 2020). Indeed to cope with the environmental stresses, the plant can reduce its growth rate or store diverse types of osmoprotectants such as glycine betaine (GB), proline, or sugars. And due to the contribution of GB to osmotic adjustment and protection of proteins and membranes from damages caused by the abiotic stresses, plentiful experiments of application of GB on its low accumulator and non-accumulator plant species have been done (Annunziata et al. 2019). In fact, GB as a quaternary ammonium compound is one of the most well-known osmoprotectants and is able to provide stress tolerance in various plant species. In their life cycles, plants can accumulate GB to regulate the disturbance of the osmotic homeostasis caused by environmental stresses such as salinity, drought, or cold stress ( $\mathrm{Xu}$ et al. 2018, Kumar et al. 2019). The roles of GB in plants under stresses have been studied in numerous plant species. Plants sprayed with GB exhibited drought stress tolerance in tomato (Ma et al. 2007), soybean (Agboma et al. 1997), turnip rape (Mäkelä et al. 1997), and rice (Farooq et al. 2008). The increase of chilling tolerance by exogenous GB in tomato (Park et al. 2006), wheat (Allard et al. 1998), and maize (Chen et al. 2000) was also reported. Indeed many studies have proven the implication of exogenous GB in preserving the photosystem II (PS II) and its capacity to increase the net photosynthetic rate and stomatal conductance during abiotic stresses (Annunziata et al. 2019, Mäkelä et al. 1999). Exogenous GB is able to inhibit the MDA and ROS over-accumulation in plants under environmental stresses and activate the expression of oxidative stress response genes (Annunziata et al. 2019). Exogenous GB has a major role in regulating the antioxidant activities in plants under drought, salinity, or cold stress (Kumar 2021a,b). Several related reports have shown the effectiveness of GB in increasing the activities of catalase (CAT), superoxide dismutase (SOD), guaiacol peroxidase (POD), or ascorbate peroxidase (APX) in various plants subjected to different abiotic stresses (Ma et al. 2006, Malekzadeh 2015, Shan et al. 2016). However, as far as we know, there is no previous research that has investigated the effect of exogenous GB on alternative oxidase activity (AOX) in plants under drought or cold stress. Furthermore, few studies have focused on the effect of exogenous GB on the woody plants, and most of them have relied on crops.

Dalbergia odorifera, also named fragrant rosewood, is an endemic species to China, which is a highlyvalued species suffering from vulnerability due to overdevelopment for wood and medicine (Li et al. 2018). Further, there is a huge lack of studies concerning this species and particularly its responses to abiotic stresses even with its high profits. Thus, the purpose of this present work was to study the responses of $D$. odorifera against drought and cold stress as affected by GB treatment. The AOX is a ubiquinol oxidase located in plant mitochondria, it can protect against unexpected inhibition of the cytochrome $c$ oxidase pathway and plays an important role in stress tolerance (Berthold et al. 2000, Mróz et al. 2015). The aim of our work was to analyze the antioxidant enzymes in
D. odorifera seedlings under drought and cold stress and investigate whether the application of exogenous GB is able to increase the AOX protein content in plants under such stresses. Indeed, in our previous study, we reported the increase in the amount of AOX protein in salt-tolerant D. odorifera seedlings (Cisse et al. 2021a) and Annunziata et al. (2019) showed the ability of exogenous GB to increase the activities of antioxidant enzymes in diverse plant species. Furthermore, the principal component analysis was used to analyze the association or dispersion of the ROS scavengers throughout the experiment.

\section{Materials and methods}

Plants and experimental design: Dalbergia odorifera $\mathrm{T}$. Chen seeds used in this experiment were collected from the plantation forest at Ledong county, Hainan Province in China ( $\left.18^{\circ} 42^{\prime} 57.91^{\prime \prime} \mathrm{N}, 108^{\circ} 52^{\prime} 18.65^{\prime \prime} \mathrm{E}\right)$ in March 2019. Sterilized and healthy seeds were selected and sown in plastic bags $(10 \times 10 \times 10 \mathrm{~cm})$, and the substrate was composed of $70 \%$ red soil and $30 \%$ sand. Each plastic bag contained 2 - 3 seeds pre-treated with hot water $\left(60^{\circ} \mathrm{C}\right)$ for $10 \mathrm{~min}$ and warm water $\left(28^{\circ} \mathrm{C}\right)$ for $10 \mathrm{~h}$. The pots were placed into an artificial incubator $Q H X-400 B H S$ III (CIMO Medical Instrument Manufacturing Co., Shanghai). The seeds were germinated under alternating temperature and humidity of $25 / 28{ }^{\circ} \mathrm{C}$ and $85 / 75 \%$ with a 12-h photoperiod and with photosynthetic photon flux density of $67.56 \mu \mathrm{mol} \mathrm{m}{ }^{-2} \mathrm{~s}^{-1}$ (Pu et al. 2021).

After 90 d (15 September - December 2019), the selected seedlings of the same size were placed in an artificial incubator (AI) and exposed to two stresses (cold and drought). For drought stress, the plants were well-watered $15 \mathrm{~h}$ before the beginning of the treatment. Indeed a low amount of water has been administered to the seedlings to a final soil water content of $40 \%$ between 2 - $3 \mathrm{~d}$. To maintain the mild drought conditions in D. odorifera seedlings the mass of the plastic bags was measured every day and clean water was added to a final water content of $40 \%$ until the final harvest point as described by Wikberg and Ögren (2007). The seedlings under chilling stress were well-watered during the experiment and placed in a growth chamber set up at $20 / 4{ }^{\circ} \mathrm{C}$ temperatures with a $16 / 8 \mathrm{~h}$ photoperiod. In each of the 5 days at the end of the $4{ }^{\circ} \mathrm{C}$ treatment, the fresh leaves in the middle were harvested, then quickly frozen in liquid $\mathrm{N}_{2}$ and stored at $-80^{\circ} \mathrm{C}$ for different biochemical parameter analyses. Well-watered plants were used as controls; two groups of seedlings (6 replicates/4 seedlings) were exposed to different conditions. The first group was placed in natural conditions (December - January) and submitted to an average day/night temperatures of $22 / 17^{\circ} \mathrm{C}(\mathrm{Nc})$. Meanwhile, the second group of seedlings was transferred to a growth chamber with day/night temperatures of $28 / 25{ }^{\circ} \mathrm{C}$ (AI). The glycine betaine $(50 \mathrm{mM})$ treatment was applied firstly on the soil $\left(1.5 \mathrm{~cm}^{3}\right)$ and then sprayed on the leaves $\left(0.5 \mathrm{~cm}^{3}\right)$ every two days (one time) according to our previous experiment (Cisse et al. 2021a). Following GB application or not, the experiment presented 7 groups 
with 24 seedlings each: 1) control (Ck): well-watered plants (AI); 2) $\mathrm{Ck}+\mathrm{GB}$ : well-watered plants $+50 \mathrm{mM}$ GB (AI); 3) Nc: well-watered plants in natural conditions; 4) drought: soil water content of $40 \%(\mathrm{AI}) ; 5)$ drought + GB: soil water content of $40 \%+50 \mathrm{mM} \mathrm{GB}(\mathrm{AI})$; 6 ) cold: 20/4 ${ }^{\circ} \mathrm{C}$ (AI); 7) cold + GB: $20 / 4{ }^{\circ} \mathrm{C}+50 \mathrm{mM}$ GB (AI). The Ck: well-watered condition (AI) was set up as a group of control for the comparison of the results in the present study since it was submitted approximately to the optimal conditions of Dalbergia odorifera growth.

Measurement of the roots membrane permeability: Cell death in root samples which indicate the loss of plasma membrane integrity was measured spectrophotometrically using the absorption of Evans blue solution (Baker and Mock 1994). The intensity of the colour is proportional to the number of dead cells. Three root ends $(1 \mathrm{~cm})$ are cut and then incubated in $0.025 \%(\mathrm{~m} / \mathrm{v})$ Evans blue solution for $30 \mathrm{~min}$. Then the roots were rinsed for $15 \mathrm{~min}$ and crushed in $800 \mathrm{~mm}^{3}$ of a solution of $50 \% \mathrm{MeOH}(\mathrm{v} / \mathrm{v})$ and $1 \%(\mathrm{~m} / \mathrm{v})$ sodium dodecyl sulphate (SDS). The homogenate was incubated in a water bath at $50{ }^{\circ} \mathrm{C}$ for $15 \mathrm{~min}$ and then centrifuged at $10000 \mathrm{~g}$ for $15 \mathrm{~min}$. The absorbance of the supernatant was read at $600 \mathrm{~nm}$.

Electrolyte leakage, water potential, and relative water content determination: Electrolyte leakage was determined according to Cisse et al (2021b) by using 5 discs from fresh leaves that were rinsed in a Petri dish containing distilled water $(\mathrm{C} 1)$. The relative conductivity was calculated by using the following formula $\mathrm{EL}=\mathrm{C} 1 \times 100 / \mathrm{C} 2$ according to Fan et al. $(2015)$ where $\mathrm{C} 2$ was conductivity of samples placed in a boiling water bath for $20 \mathrm{~min}$ and then cooled to room temperature. The dew point water potential (DWP) was determined with a DWP meter (WP4C, Gene Company, America) in accordance with the manufacturer's instructions. The relative water content was determined in fresh leaves as described by Yang et al. (2010).

The content of chlorophylls and carotenoids was determined by the colorimetric method. Approximately $0.2 \mathrm{~g}$ of fresh leaves was ground in $10 \mathrm{~cm}^{3}$ of $80 \%(\mathrm{v} / \mathrm{v})$ acetone, then the extract was centrifuged at $10000 \mathrm{~g}$ and $4{ }^{\circ} \mathrm{C}$ for $10 \mathrm{~min}$, and the supernatant was used for spectrophotometer readings at 663, 646, and $470 \mathrm{~nm}$ The content of pigments in each sample was calculated according to Lichtenthaler and Wellburn (1983).

Determination of malondialdehyde (MDA) and hydrogen peroxide: MDA content was determined by the method described by Yang et al. (2010) with some modifications. About $200 \mathrm{mg}$ of sample was homogenized in $5 \mathrm{~cm}^{3}$ of $5 \%(\mathrm{~m} / \mathrm{v})$ trichloroacetic acid (TCA) and then centrifuged at $10000 \mathrm{~g}$ for $10 \mathrm{~min}$. Then $4 \mathrm{~cm}^{3}$ of $20 \%(\mathrm{~m} / \mathrm{v})$ TCA containing $0.5 \%(\mathrm{~m} / \mathrm{v})$ thiobarbituric acid (TBA) and $1 \mathrm{~cm}^{3}$ of the supernatant were incubated at $95{ }^{\circ} \mathrm{C}$ for $30 \mathrm{~min}$, cooled on ice, centrifuged at $10000 \mathrm{~g}$ for $15 \mathrm{~min}$ and absorbance was read at $532 \mathrm{~nm}$ and the content of MDA was expressed in [nmol g-1 (f.m.)].
The hydrogen peroxide was quantified as described by Yang et al. (2010) with some modifications. Fresh leaves samples (200 mg) were grounded and homogenized in $0.1 \%$ trichloroacetic acid $(\mathrm{m} / \mathrm{v})$ then centrifuged at $10000 \mathrm{~g}$ for $15 \mathrm{~min}$. A supernatant was incubated in the presence of potassium iodide added to a $10 \mathrm{mM}$ buffer solution. The absorbance was determined at $390 \mathrm{~nm}$, and the content of $\mathrm{H}_{2} \mathrm{O}_{2}$ was estimated via a standard curve.

Measurement of proline, saccharides, and total phenols: Proline content was determined by the colorimetric assay as described by Bates et al. (1973) with some modifications according to Cisse et al. (2021b).

Content of saccharides was estimated by the anthrone method ( $0.2 \%$ anthrone) according to Yemm and Willis (1954) with some modifications following the description of Cisse et al. (2021b). The absorbance was read at $627 \mathrm{~nm}$ and a standard curve was used to calculate the content of the saccharides in each sample and expressed in $\left[\mu \mathrm{g} \mathrm{g}^{-1}\right.$ (f.m.)].

Phenolic compounds were determined according to the Folin-Ciocâlteu reagent method (Swain and Hillis 1959). The phenolic compounds were extracted using $80 \%$ (v/v) ethanol at $80{ }^{\circ} \mathrm{C}$ for $15 \mathrm{~min}$ and then the extract was concentrated by evaporating the ethanol. The residue was dissolved in $20 \mathrm{~cm}^{3}$ of distilled water and to $1 \mathrm{~cm}^{3}$ of aliquot, it was added $7.5 \mathrm{~cm}^{3}$ of distilled water and $0.5 \mathrm{~cm}^{3}$ of Folin's reagent. After $3 \mathrm{~min}, 1 \mathrm{~cm}^{3}$ of saturated $\mathrm{Na}_{2} \mathrm{CO}_{3}$ solution $(40 \%)$ was added to the tube and after $1 \mathrm{~h}$ absorbance was read at $725 \mathrm{~nm}$. The content of phenols was determined according to the following formula: $\mathrm{A}_{725} \times$ initial volume/fresh mass.

Measurement of proteins and thiols: Soluble protein content was quantified by the Bradford method (1976). The absorbance was recorded at $595 \mathrm{~nm}$ and the content of protein was determined by using a standard curve.

Thiols react with 5,5'-dithiobis (2-nitrobenzoic acid) DTNB to form 2-nitro-5-thiobenzoic acid (TNB), which turns the colour of the solution to yellow in an alkaline medium and absorbance was read at $412 \mathrm{~nm}$ (Ellman 1959). The content of thiols was assayed and determined according to Cisse et al. (2021a).

Measurement of antioxidant enzyme activities and AOX protein content: Peroxidase (POD) activity was quantified following the method described by Fielding and Hall (1978) with some modifications. An amount of $0.5 \mathrm{~g}$ of fresh leaves was washed with distilled water, weighed, and triturated in a mortar at $4{ }^{\circ} \mathrm{C}$ by using $5 \mathrm{~cm}^{3}$ of $0.1 \mathrm{M}$ phosphate buffer solution $(\mathrm{pH} 7.0)$ containing $0.1 \mathrm{mM}$ EDTA, $0.1 \mathrm{mM}$ ascorbate, and $1 \%$ polyvinylpolypyrrolidone (PVPP). The sample was centrifuged at $10000 \mathrm{~g}$ and $4{ }^{\circ} \mathrm{C}$ for $15 \mathrm{~min}$ and the supernatant was used to assay the enzymatic activity of POD. The increment in absorption induced by guaiacol oxidation by $\mathrm{H}_{2} \mathrm{O}_{2}$ was measured at $470 \mathrm{~nm}$. Catalase (CAT) activity was determined by colorimetric assay at $240 \mathrm{~nm}$ by measuring the decomposition of $\mathrm{H}_{2} \mathrm{O}_{2}$ according to the procedure described by Aebi (1984) with 
some modifications. A sample of $0.3 \mathrm{~g}$ fresh leaves was homogenized with $0.1 \mathrm{mM}$ potassium phosphate buffer (pH 7.8) on ice. The extract was centrifuged at $10000 \mathrm{~g}$ and $4{ }^{\circ} \mathrm{C}$ for $15 \mathrm{~min}$ and then the supernatants were used to assay the CAT activities.

ELISA kit (JL22749 Plant AOX ELISA KIT; Jianglai Industrial Company, Shanghai, China) was used to determine the content of AOX protein in plant leaves according to Cisse et al. (2021a). A standard curve was used to estimate the content of AOX protein which was expressed in $\left[\mathrm{ng} \mathrm{g}^{-1}\right.$ (f.m.)]. The protein formation of AOX does not directly determine the activity of the enzyme, but the increase of AOX protein is related to the AOX enzyme activity (Saha et al. 2016).

Statistical analyses: The results were expressed as mean \pm standard error, and Graph Pad Prism 9.0.0 software was used to draw the graphs and analyze the data. All data were subjected to analysis of variance for a factorial experiment in a completely randomized design. A two way ANOVA Tukey's HSD (honestly significant difference) test was used to determine the significance of our data at $P \leq 0.05$. The principal component analysis (PCA) was performed by Graph Pad Prism 9.0.0.

\section{Results}

The group of seedlings submitted to optimal conditions represents the control group in this current experiment (Ck). Drought and cold stress significantly decreased the plant height, the diameter of the stem, and leaf thickness in $D$. odorifera seedlings (Table 1 Suppl.). The roots length was slightly diminished by the drought and cold stress. Generally, under both stresses, the seedlings subjected to GB treatment exhibited a significant increase of root length, plant height increment and diameter of the stem in comparison with the cold or drought-stressed seedlings (Table 1 Suppl. and Fig. 1 Suppl.). The leaf thickness was only increased $(45.16 \%)$ by GB under drought stress compared to only drought-stressed seedlings. The seedlings that were subjected to drought without GB treatment, showed a significant decrease of RWC (22.85\%), and a decrease $(22.85 \%)$ of WP (Table 1 Suppl.). Meanwhile, GB did not influence significantly the RWC and WP in seedlings under cold stress. Fig. 2 Suppl. showed that after $2 \mathrm{~d}$ the seedlings submitted to cold stress exhibited injuries that can lead to the death of the plant after $7 \mathrm{~d}$ (Fig. 3 Suppl.); however, the cold-stressed plants treated by GB showed fewer injuries and stay alive beyond $7 \mathrm{~d}$. Under non-stress conditions, the seedlings subjected to optimal conditions $(\mathrm{Ck})$ showed better growth parameters compared to those under natural conditions (representing cold season). The addition of GB did not significantly increase the growth traits under non-stressed conditions.

The total chlorophyll $\left(\mathrm{Chl}_{a+b}\right)$ content in $D$. odorifera leaves was strongly decreased by $80 \%$ in the cold and drought treatments, compared to the control group $(\mathrm{Ck}$, $P \leq 0.001)$. The carotenoid (Car) content in cold-stressed seedlings was significantly higher $(19.35 \%)$ compared to drought-stressed seedlings. Under drought or cold stress, the $\mathrm{Chl}$ content in seedlings was slightly increased by the GB treatment, but it was not significantly different from that without GB treatment. The Car content in coldstressed seedlings was higher by $6.06 \%$ compared to the GB cold-stressed seedlings (Table 1). Under non-stress conditions, the content of photosynthetic pigments was similar in $\mathrm{Ck}, \mathrm{Ck}+\mathrm{GB}$ and $\mathrm{Nc}$ groups.

Table 1 also shows the effect of drought and cold stress treatment combined with GB or not on root cell integrity. The absorption of the Evans blue solution was significantly higher in drought $(63.02 \%)$ and cold-stressed $(62.03 \%)$ seedlings compared to $\mathrm{Ck}$. GB treatment reduced by $18.53 \%$ the absorption of the blue Evans solution in the GB cold-stressed seedlings compared to the only cold-stressed seedlings. Under normal conditions, GB treatment improved significantly the membranes of the roots compared to $\mathrm{Ck}$ and $\mathrm{Nc}$ groups based on the lower absorption of the blue Evans solution in $\mathrm{Ck}+\mathrm{GB}$.

Electrolyte leakage (EL) is an important indicator related to leaf senescence and cell membrane deterioration. The results of the experiment confirm that the drought and cold stress applied to $D$. odorifera seedlings increased significantly the EL by 31.81 and $52 \%$, respectively, compared to the control (Table 1). Moreover, under cold

Table 1. Variations of content of chlorophylls (Chl) and carotenoids (Car), Evans blue absorption, electrolyte leakage (EL), content of phenolic compounds and thiols in Dalbergia odorifera leaves under drought and cold stresses and treated by GB, (for more detail see Materials and methods). Means $\pm \mathrm{SEs}, n=6$; different letters indicate significant differences between treatments $(P<0.05)$ based on Tukey's multiple comparison tests.

\begin{tabular}{|c|c|c|c|c|c|c|c|}
\hline Treatments & $\begin{array}{l}\mathrm{Chl}_{a} \\
{\left[\mathrm{mg} \mathrm{g}^{-1} \text { (f.m.)] }\right.}\end{array}$ & $\begin{array}{l}\mathrm{Chl}_{b} \\
{\left[\mathrm{mg} \mathrm{g}^{-1} \text { (f.m.) }\right]}\end{array}$ & $\begin{array}{l}\mathrm{Car} \\
{\left[\mathrm{mg} \mathrm{g}^{-1} \text { (f.m.) }\right]}\end{array}$ & $\begin{array}{l}\text { Evans blue } \\
{[\%]}\end{array}$ & EL [\%] & $\begin{array}{l}\text { Phenols } \\
{\left[\mathrm{mg} \mathrm{g}^{-1} \text { (f.m.) }\right]}\end{array}$ & $\begin{array}{l}\text { Thiols } \\
{\left[\mathrm{mg} \mathrm{g}^{-1} \text { (f.m.)] }\right.}\end{array}$ \\
\hline $\mathrm{Ck}$ & $1.1 \pm 0.17 \mathrm{a}$ & $0.32 \pm 0.05 \mathrm{ab}$ & $0.28 \pm 0.03 \mathrm{a}$ & $23.8 \pm 7.6 \mathrm{bc}$ & $14.8 \pm 4.5 \mathrm{~b}$ & $21.6 \pm 0.89 \mathrm{~b}$ & $1.02 \pm 0.02 \mathrm{c}$ \\
\hline $\mathrm{Ck}+\mathrm{GB}$ & $1.3 \pm 0.23 \mathrm{a}$ & $0.31 \pm 0.11 \mathrm{ab}$ & $0.21 \pm 0.05 \mathrm{ab}$ & $14.6 \pm 3.9 \mathrm{c}$ & $16.5 \pm 3.4 \mathrm{~b}$ & $26.2 \pm 1.67 \mathrm{a}$ & $1.21 \pm 0.07 \mathrm{~b}$ \\
\hline $\mathrm{Nc}$ & $1.1 \pm 0.26 \mathrm{a}$ & $0.41 \pm 0.10 \mathrm{a}$ & $0.18 \pm 0.02 \mathrm{abc}$ & $32.4 \pm 2.8 \mathrm{~b}$ & $15.6 \pm 5.3 \mathrm{~b}$ & $18.3 \pm 0.83 \mathrm{cde}$ & $1.05 \pm 0.02 \mathrm{c}$ \\
\hline Drought & $0.5 \pm 0.08 \mathrm{~b}$ & $0.16 \pm 0.09 \mathrm{c}$ & $0.14 \pm 0.01 \mathrm{c}$ & $45.7 \pm 10.4 \mathrm{a}$ & $20.4 \pm 2.8 \mathrm{ab}$ & $19.7 \pm 1.61 \mathrm{bcd}$ & $1.24 \pm 0.08 \mathrm{~b}$ \\
\hline Drought + GB & $0.6 \pm 0.14 b$ & $0.17 \pm 0.04 \mathrm{c}$ & $0.14 \pm 0.02 \mathrm{c}$ & $45.2 \pm 6.3 \mathrm{a}$ & $19.8 \pm 1.7 \mathrm{ab}$ & $20.4 \pm 0.19 b c$ & $1.54 \pm 0.02 \mathrm{a}$ \\
\hline Cold & $0.4 \pm 0.13 b$ & $0.18 \pm 0.11 \mathrm{c}$ & $0.17 \pm 0.03 \mathrm{abc}$ & $33.6 \pm 8.7 \mathrm{ab}$ & $25.2 \pm 6.1 \mathrm{a}$ & $17.3 \pm 1.42 \mathrm{e}$ & $1.04 \pm 0.06 \mathrm{c}$ \\
\hline Cold+GB & $0.7 \pm 0.10 \mathrm{~b}$ & $0.20 \pm 0.08 b c$ & $0.16 \pm 0.01 \mathrm{bc}$ & $27.9 \pm 3.5 b$ & $21.9 \pm 2.8 \mathrm{ab}$ & $17.7 \pm 0.37 \mathrm{de}$ & $0.97 \pm 0.03 \mathrm{c}$ \\
\hline
\end{tabular}




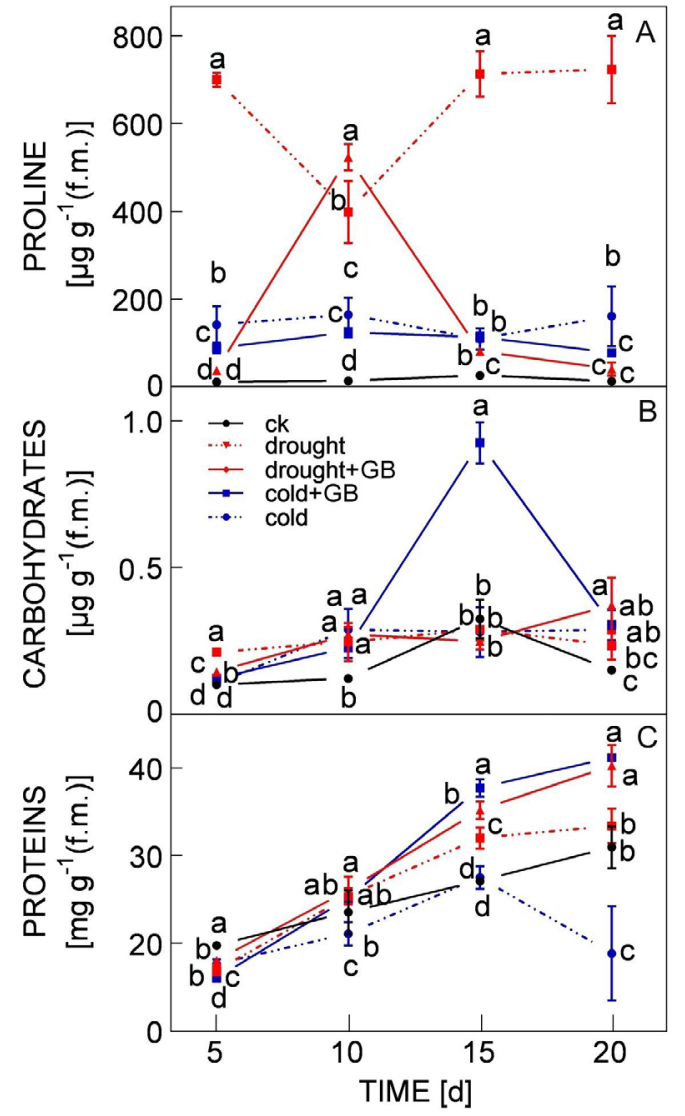

Fig. 1. Effects of glycine betaine (GB) on the content of proline $(A)$, sugars $(B)$ and proteins $(C)$ in Dalbergia odorifera under drought and cold stress. Means \pm SEs, $n=6$, different letters indicate significant differences according to Tukey's multiple comparison test $(P<0.05)$. ck - control.

stress, GB treatment reduced significantly (14.01\%) the EL in GB cold-stressed seedlings compared to the seedlings that were not subjected to GB treatment.

Our data in Table 1 show a significant increase in the content of phenolic compounds by GB treatment in D. odorifera seedlings under stress. However, the amount of phenolic compounds was strongly higher $(14.17 \%)$ in seedlings subjected to drought than in those under cold stress. Moreover, the thiols content in drought-stressed seedlings was significantly higher $(17.54 \%)$ than in cold-stressed plants. The content of thiols did not show significant differences between $\mathrm{Ck}$ seedlings and those submitted to cold stress. Without stress, the thiols and phenolic compounds were significantly enhanced by GB treatment in D. odorifera seedlings compared to $\mathrm{Ck}$ and Nc groups.

The content of sugars and proline of D. odorifera leaves was higher in seedlings under drought or cold stress compared to the $\mathrm{Ck}$ (Fig. 1A,B). Under drought stress, GB treatment mostly decreased the proline content in $D$. odorifera seedlings (Fig. 1A). Meanwhile, a comparison of cold-stressed and GB cold-stressed seedlings show a slight difference. Furthermore, the content of proline in $D$. odorifera leaves was strongly higher in seedlings

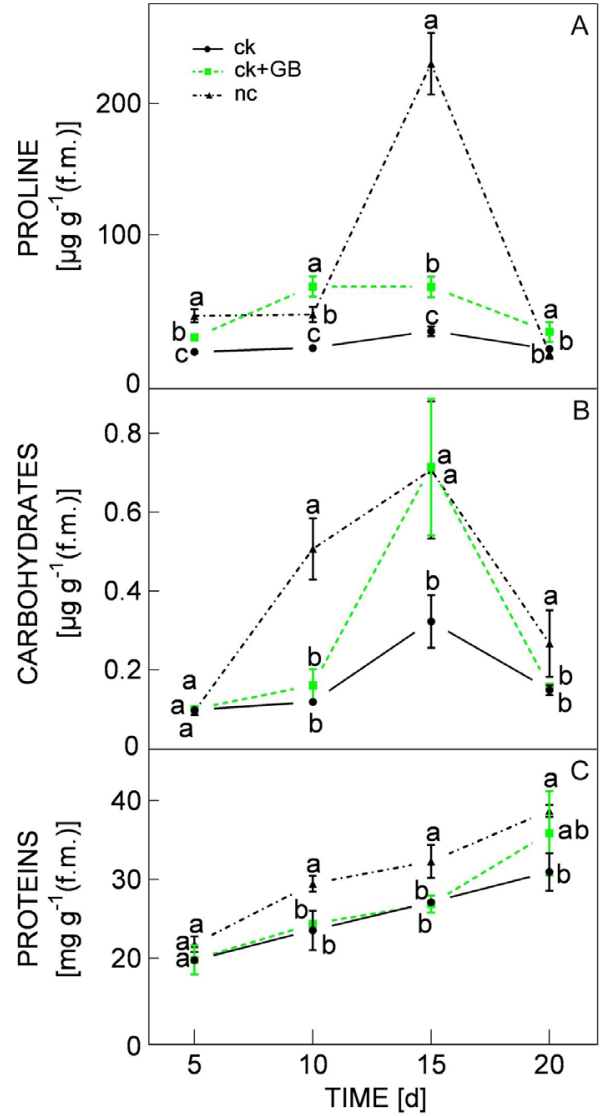

Fig. 2. Effects of glycine betaine (GB) on the content of proline $(A)$, sugars $(B)$ and proteins $(C)$ in Dalbergia odorifera without stress. Means \pm SEs, $n=6$, different letters indicate significant differences according to Tukey's multiple comparison test $(P<0.05)$. ck - control, nc - natural conditions.

that were subjected to drought compared to those under cold stress. Nonetheless, at day 10 the proline content strongly increased by $171.68 \%$ in seedlings subjected to $\mathrm{GB}$ treatment under drought stress as compared to the drought-stressed seedlings. The results also showed that GB treatment decreased significantly $(37.97 \%)$ the sugar content in seedlings under drought compared to the drought-stressed seedlings, however, the sugar content was increased $(15.96 \%)$ in plants treated by GB under chilling stress at day 5 (Fig. 1B). However, under drought stress, the GB treatment enhanced significantly the sugar content in D. odorifera leaves only after day 20 . Generally, under normal conditions, GB treatment $(\mathrm{Ck}+\mathrm{GB})$ increased slightly sugar content in seedlings compared to the $\mathrm{Ck}$ group; meanwhile, it increased significantly the proline content in $D$. odorifera seedlings (Fig. $2 A, B$ ). The soluble protein content of $D$. odorifera seedling leaves was significantly increased by GB treatment under drought and cold stress (Fig. 1C). The seedlings under cold stress without GB treatment exhibited a significant decrease in protein content during the experiment compared to drought-stressed seedlings. The fluctuation of proline and sugars in seedlings subjected to natural conditions (cold season) were similar to those in $\mathrm{Ck}+\mathrm{GB}$ group at 


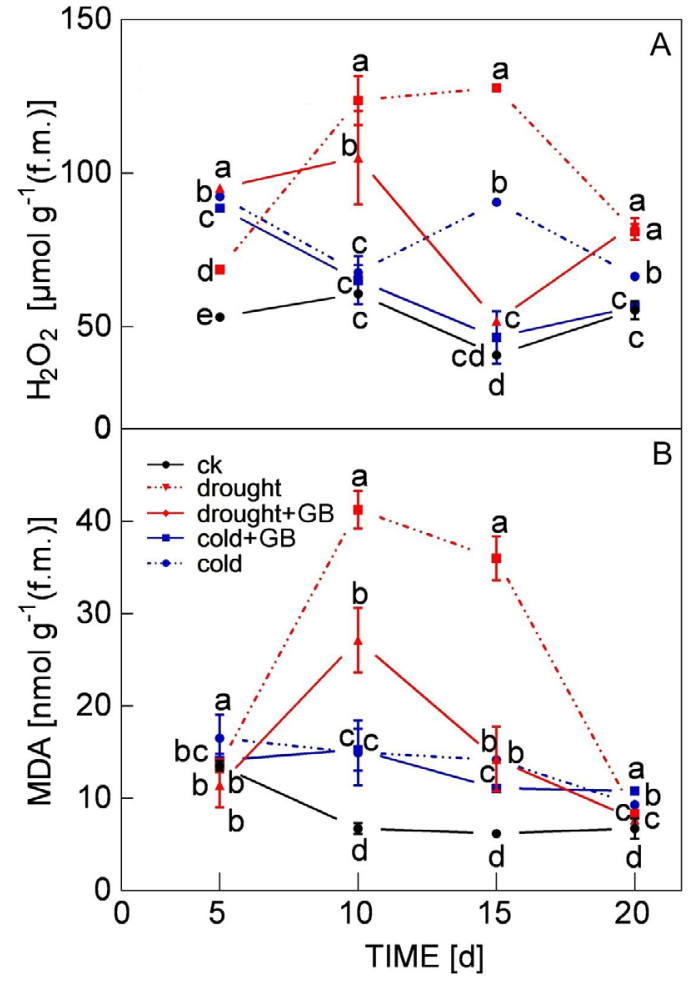

Fig. 3. Content of $\mathrm{H}_{2} \mathrm{O}_{2}(A)$ and MDA $(B)$ in Dalbergia odorifera seedlings subjected to glycine betaine (GB) treatment under drought and cold stress. Means \pm SEs, $n=6$, different letters indicate significant differences according to Tukey's multiple comparison test $(P<0.05)$. ck - control.

different times (Fig. 2A,B). Meanwhile, without stress, the protein content was higher in seedlings exposed to natural conditions compared to $\mathrm{Ck}$ and $\mathrm{Ck}+\mathrm{GB}$ groups, while the GB increased significantly the protein content at day 20 compared to $\mathrm{Ck}$ (Fig. 2C).

As shown in Fig. 3, during the cold and drought treatment, $\mathrm{H}_{2} \mathrm{O}_{2}$ and MDA content in D. odorifera seedlings were significantly higher compared to $\mathrm{Ck}$. The $\mathrm{H}_{2} \mathrm{O}_{2}$ content in $D$. odorifera seedlings was strongly lower at days 5 and 15 in those grown under natural conditions compared to $\mathrm{Ck}$ and $\mathrm{Ck}+\mathrm{GB}$ (Fig. 4A). Generally, after $10 \mathrm{~d}$ the drought-stressed seedlings showed a significant increase $(75.55 \%$ at day 15 and $17.97 \%$ at day 20$)$ in $\mathrm{H}_{2} \mathrm{O}_{2}$ content as compared with the seedlings that were submitted to chilling stress. Moreover, during both stresses, the application of GB treatment decreased strongly the content of $\mathrm{H}_{2} \mathrm{O}_{2}$ in $D$. odorifera seedlings compared to the stressed seedlings (Fig. 3A).

During this experiment, the GB treatment decreased strongly the MDA content in drought-stressed seedlings between $10(45 \%)$ to $15(62.28 \%)$ days (Fig. 3B). Meanwhile, under cold stress, the GB treatment reduced slightly the MDA content in D. odorifera seedlings (day 15). Without stress, the MDA content was similar in the control groups, but at day 15 the MDA content was significantly higher in seedlings subjected to GB treatment. During the treatments, the seedlings that were exposed to natural conditions showed an enhancement in MDA content compared to $\mathrm{Ck}$ and $\mathrm{Ck}+\mathrm{GB}$ at days 5, 10,

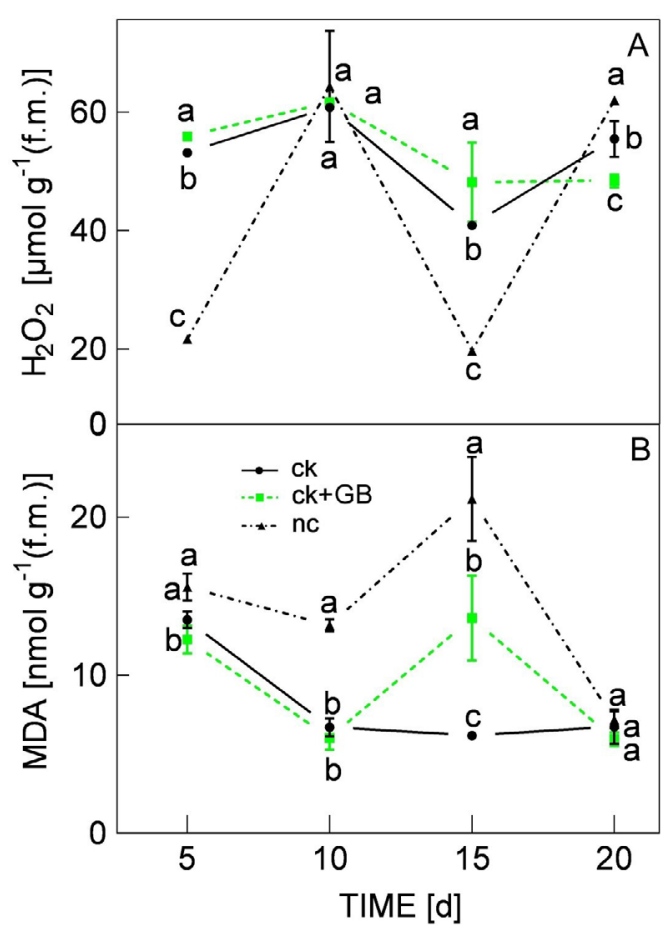

Fig. 4. Content of $\mathrm{H}_{2} \mathrm{O}_{2}(A)$ and MDA $(B)$ in Dalbergia odorifera seedlings subjected to glycine betaine (GB) treatment without stress. Means \pm SEs, $n=6$, different letters indicate significant differences according to Tukey's multiple comparison test $(P<0.05)$. ck - control, nc - natural conditions.

and 15 (Fig. 4B).

The AOX protein content was considerably higher in seedlings under drought (129 and 171. 52\%) or cold $(71.85$ and $163.25 \%)$ stress and GB treatment at days 5 and 10 compared to $\mathrm{Ck}$. Furthermore, under drought stress the GB treatment decreased significantly the AOX protein content, except for day 20 where the AOX protein content was greater in GB drought-stressed seedlings compared to only drought-stressed seedlings (Fig. $5 A$ ). Simultaneously, the GB treatment increased significantly the AOX protein content in seedlings under cold stress. Mostly, the activities of antioxidant enzymes in $D$. odorifera seedlings were significantly higher between days 5 and 10 in the cold and drought-stressed seedlings compared to $\mathrm{Ck}$ ( $P$ $<0.001$, Fig. $5 B, C)$. Generally, the peroxidase activity increased significantly with GB treatment under both stresses throughout the experiment (days 5, 10, 15, and 20) compared to the other treatments (Fig. 5C). Moreover, during the experiment, the POD activity was similar in seedlings under drought and cold stress without GB treatment. Furthermore, under cold stress, the seedlings subjected to GB treatment increased strongly the CAT activity at day $5(9.21 \%), 10(40.16 \%), 15(21.73 \%)$, and day $20(50.06 \%)$ compared to the cold-stressed seedlings. At day 20 in seedlings under cold stress without GB treatment, the CAT activity was strongly lower than in the $\mathrm{Ck}$ (Fig. 5B). Moreover, besides cold or drought stress the GB treatment increased strongly the AOX content in D. odorifera seedlings only at day 10 compared to $\mathrm{Ck}$. 


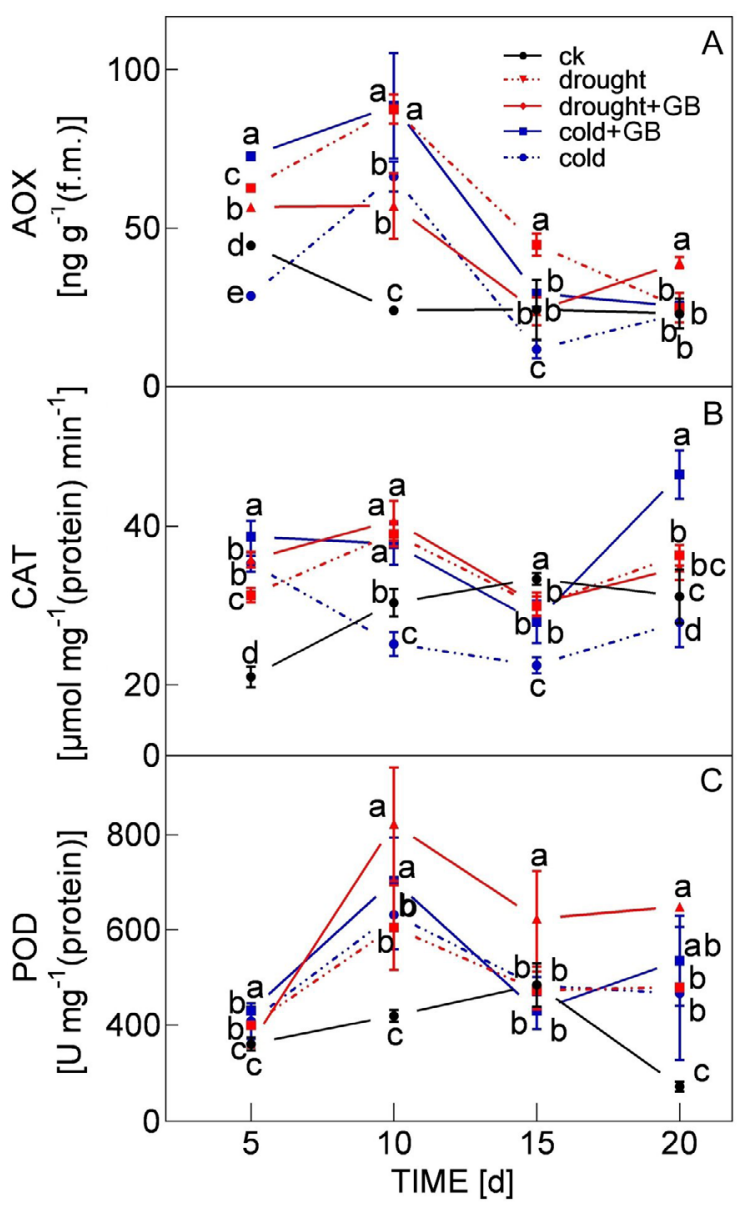

Fig. 5. Content of AOX protein $(A)$ and activities of CAT $(B)$ and POD $(C)$ in Dalbergia odorifera seedlings treated with glycine betaine (GB) and under drought or cold stress. Means \pm SEs, $n$ $=6$, different letters indicate significant differences according to Tukey's multiple comparison test $(P<0.05)$. ck - control.

The seedlings that were exposed to natural conditions showed a significant increase in the AOX content at day 15 (Fig. 6A). Furthermore, under non-stress conditions the CAT activity was significantly enhanced in $\mathrm{Ck}+\mathrm{GB}$ seedlings throughout the experiment compared to the $\mathrm{Ck}$ group, moreover, the POD activity was increased at days 15 and 20 by GB treatment (Fig. $6 B, C$ ).

The assessment of the correlation between different measured parameters has been done by principal component analysis. The total cumulative proportion of variance of the PC1 and PC2 factors corresponded to $62.44 \%$, and their eigenvalues were higher than 1 . The proportion of variance of the first two principal components was 42.97 and $19.47 \%$, respectively. The heat map and loadings plot based on PC1 and PC2 was used to understand the data structure and measured parameters associations (Fig. 4 Suppl.). Fig. 5 Suppl. showed a strong dissociation between thiols and proteins; and a significant association among $\mathrm{H}_{2} \mathrm{O}_{2}$ and root membrane permeability; POD, EL, sugars, and MDA; between root height, water potential, water content, and diameter of stem; between the photosynthetic pigments at the end of the experiment (day 20). The association among AOX, POD, and CAT was not strong as indicated

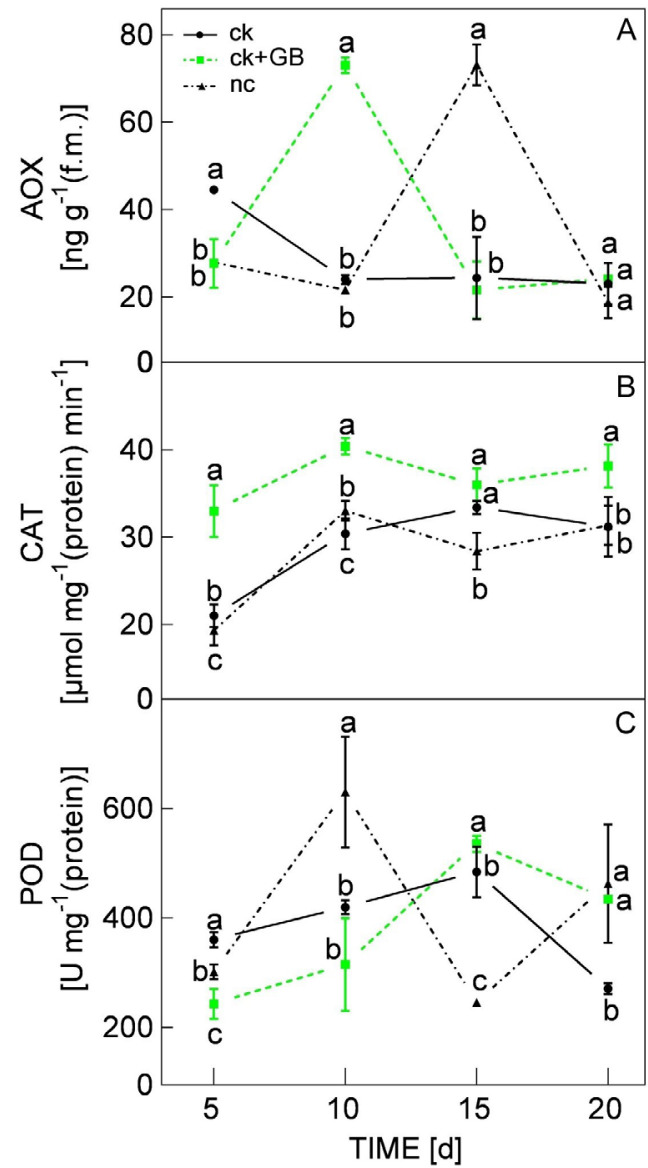

Fig. 6. Content of AOX protein $(A)$ and activities of CAT $(B)$ and POD $(C)$ in Dalbergia odorifera seedlings treated with glycine betaine (GB) without stress. Means \pm SEs, $n=6$, different letters indicate significant differences according to Tukey's multiple comparison test $(P<0.05)$. ck - control, nc - natural conditions.

by the angles between their vectors. There were two major groups of association following Fig. 5 Suppl.; the first was related to $\mathrm{Chl} a+b, \mathrm{Chl} a, \mathrm{Chl} b$, and Car. The second group belonged to growth traits (diameters of stem and root length), WP, and WC. Fig. 6 Suppl. displayed the way that different parameters related to oxidative stress are associated through the duration of the present experiment. It showed a strong association between POD and AOX; among AOX and CAT based on the small angle of their vectors (at days 5 and 10, respectively). The association between the two osmoprotectants (proline and sugars) was strong at day 5 (Fig. $6 A$ Suppl.). Indeed, the association between the different measured parameters responsible for the ROS scavenging split up following the duration of the experiment (Fig. 6C,D Suppl.).

\section{Discussion}

The evaluation of the effects of abiotic stresses on plants can be based on their effects on water status, growth parameters, antioxidant enzyme activities, photosynthesis, and other parameters. The results of the present study 


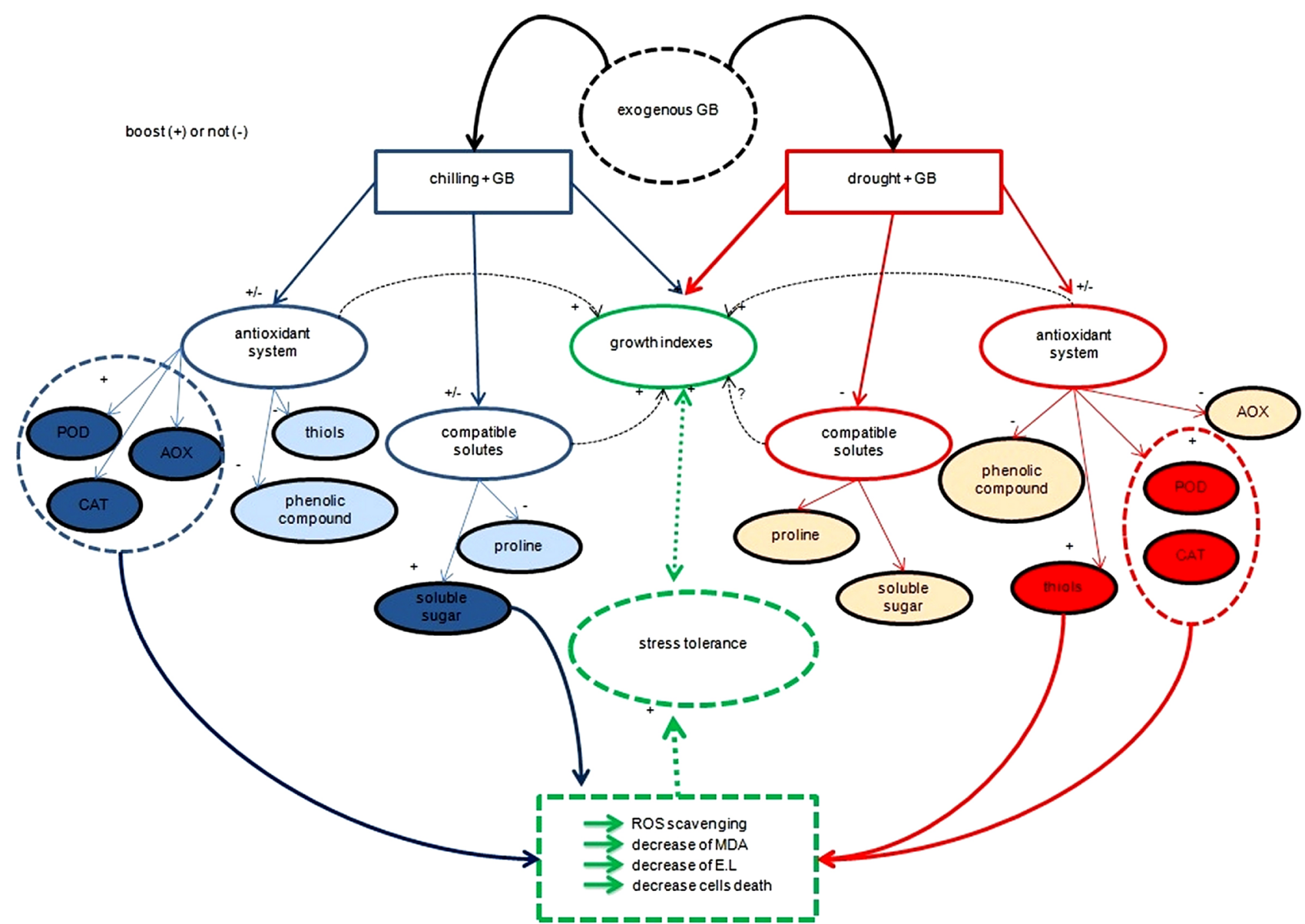

Fig. 7. A designed model showing different pathways that exogenous GB utilizes to enhance the drought and cold tolerance in Dalbergia odorifera. AOX - alternative oxidase, CAT - catalase, EL - electrolyte leakage, GB - glycine betaine, MDA - malondialdehyde, POD peroxidase, ROS - reactive oxygen species.

confirmed the negative effects of drought and cold stresses on plant growth as also mentioned by Ritonga and Chen (2020). Nonetheless, GB treatment increased significantly the growth parameters in $D$. odorifera seedlings under drought and cold stresses. Indeed, GB can improve the growth and survival of plants counterbalancing metabolic dysfunctions caused by drought or chilling (Annunziata et al. 2019). Moreover, Malekzadeh (2015) reported the positive effect of GB treatment on growth traits in the soybean plant. Drought or cold stress can decrease the absorption of water that is followed by stomata closure (Sharma et al. 2020). Furthermore, numerous studies concerning low accumulators and non-accumulators of GB proved the beneficial effect of GB on photosynthetic pigments and on preserving the PS II function (Khan et al. 2014, Tian et al. 2017). The results of this work showed better photosynthetic pigments accumulation in seedlings treated with GB under drought and cold stress. Based on the previous study, that phenomenon can be explained by the fact that GB application to leaves can permit the translocation of GB to the meristematic tissues, particularly to flower buds and shoot apices, and than interact with photosynthesis apparatus (Mäkelä et al. 1996, Park et al. 2006, Annunziata et al. 2019).
Moreover in this study exogenous GB relieved drought and cold stress damage to cell membranes by reducing the electrolyte leakage or roots membrane permeability in $D$. odorifera seedlings. However, the decrease of those two indexes was not significant under drought stress. Indeed, exogenous GB reduced significantly EL and root membrane permeability provoked by cold and drought stress in D. odorifera seedlings. The increase of the root membrane permeability and electrolyte leakage in stressed seedlings occurred together with a high accumulation of $\mathrm{H}_{2} \mathrm{O}_{2}$. Indeed, $\mathrm{GB}$ can decrease the EL and root membrane permeability via its ability to scavenge directly the ROS or might interact with the efflux of various ions involved in membrane stability under stress, thus reducing loss of membrane integrity and membrane damages.

The lipid peroxidation was reduced by GB treatment, particularly under drought stress. Meanwhile, during the experiment, the seedlings submitted to cold stress showed a slightly decreased MDA content. Indeed, the accumulation of GB in plant cells can adjust the disturbance of osmotic homeostasis caused by abiotic stresses such as cold or drought stress ( $\mathrm{Xu}$ et al. 2018). Also, Chen and Murata (2008) suggested that one of the roles of GB might be to protect cell membranes. Furthermore, 
the accumulation of GB in the pepper plant contributed to the protection of membrane damages caused by water deficit (Escalante-Magaña et al. 2019). Drought and cold as abiotic stresses can trigger oxidative stress in plants that is due to overproduction and accumulation of ROS. The accumulation of $\mathrm{H}_{2} \mathrm{O}_{2}$ in the plant can provoke severe damages in cell components and cause their dysfunction (Demidchik 2015, Staniak et al. 2020). The results showed a significant decrease of $\mathrm{H}_{2} \mathrm{O}_{2}$ in $D$. odorifera seedlings by GB treatment under abiotic stresses during our experiments. Previous studies showed the positive effect of GB treatment on $\mathrm{H}_{2} \mathrm{O}_{2}$ scavenging in diverse plants such as rice (Demiral and Türkan 2004) or tobacco plant (Banu et al. 2010) under abiotic stresses. Among ROS, the choice of $\mathrm{H}_{2} \mathrm{O}_{2}$ lies in the fact that $\mathrm{H}_{2} \mathrm{O}_{2}$ is more stable than the others (Sies 1993). The results suggested that GB treatment scavenge directly the ROS in D. odorifera plant cells to relieve the damages caused by the oxidative stress. To cope with oxidase stress caused by abiotic stresses, plants can use diverse pathways to counteract the overaccumulation of ROS in plant cells. Under abiotic stresses, plants can accumulate osmolytes such as proline or sugars to counterbalance the disturbed homeostasis provoked by abiotic stress and maybe directly scavenge the ROS (Sharma et al. 2019). The results showed that GB can increase the sugar content under chilling stress and also in controls. Different studies reported a positive correlation between soluble sugar content and exogenous GB in plants under abiotic stress. The increased amount of photosynthetic pigments in this present study might explain the positive effect of GB on the biosynthesis of sugars as described by Osman (2015). Indeed, the application of GB in leaves of Pisum sativum under drought stress increased the growth traits and the content of photosynthates (Osman 2015).

The proline content did not increase in $D$. odorifera seedlings by GB treatment under chilling or drought stress. Thus, the results suggested that exogenous GB might regulate the ROS scavenging and cell homeostasis in $D$. odorifera by regulating the compatible solutes. The accumulation of exogenous GB in plant cells might provoke the regulation of proline and sugar content in D. odorifera leaves under abiotic stresses. Indeed, as an osmolyte, GB has the same function similarly to proline or sugars (Sharma et al. 2019). Moreover, this present work confirmed the report of Yancey (1994) that related a protective role of $\mathrm{GB}$ in plants protein content under abiotic stresses. The seedlings that were submitted to GB treatment exhibited an enhancement in protein content.

Furthermore against oxidative stress, the plant can accumulate diverse antioxidants such as the phenolic compounds or the thiols which can play a major role to relieve the damages caused by environmental stresses (Zagorchev et al. 2013, Varela et al. 2016). Indeed it was also reported that the phenols own an antioxidant activity which can scavenge the ROS under abiotic stress in Hypericum pruinatum (Caliskan et al. 2017). In the present work, the thiols were significantly more involved in providing stress tolerance in $D$. odorifera seedlings compared to the phenolic compounds and particularly during drought stress. Furthermore, the other component of the antioxidant machinery has a major role to scavenge ROS (Das and Roychoudhury 2014). These studies focused on Glycine max (Malekzadeh 2015) or wheat plants (Ma et al. 2006, Raza et al. 2007, 2014). Moreover, exogenous GB provides chilling tolerance in Prunus persica by improving the antioxidant activities (Shan et al. 2016). Nonetheless, it is important to notice a lack of information about the effect of GB on AOX protein content. There is no study that focused on the effect of exogenous GB in plants AOX protein content under abiotic stresses. Despite the major role that AOX play against environmental stresses. In the present study, GB showed its ability to increase the CAT, POD activities and AOX protein content in $D$. odorifera seedlings. However, under drought stress, GB treatment did not increase the AOX protein content. But under cold stress, exogenous GB promotes the AOX protein content rather than POD activity (Fig. 7). The present work demonstrated how exogenous GB alleviated drought and cold stress in D. odorifera seedlings (Fig. 7).

\section{Conclusions}

Abiotic stresses are able to induce a series of physiological dysfunctions in woody plants. To cope with environmental stresses in a plant, it is widely considered to use exogenous glycine betaine for conferring abiotic stress tolerance to diverse plant species. The present findings confirmed that the exogenous glycine betaine promoted efficiently drought and chilling tolerance in a woody plant $D$. odorifera. However, most theories or studies about the influence of exogenous GB in plants are focused on POD, CAT, and SOD. This study proves the involvement of exogenous glycine betaine in the regulation of the plant AOX protein content during environmental stresses. Furthermore, exogenous GB provides abiotic stress tolerance in different pathways.

\section{References}

Aebi, H.: Catalase in vitro. - Method. Enzymol. 105: 121-126, 1984.

Agboma, P.C., Sinclair, T.R., Jokinen, K., Peltonen-Sainio, P., Pehu, E.: An evaluation of the effect of exogenous glycinebetaine on the growth and yield of soybean: timing of application, watering regimes and cultivars. - Field Crops Res. 54: 51-64, 1997.

Allard, F., Houde, M., Krol, M., Ivanov, A., Huner, N.P.A., Sarhan, F.: Betaine improves freezing tolerance in wheat. Plant Cell Physiol. 39: 1194-1202, 1998.

Annunziata, M.G., Ciarmiello, L.F., Woodrow, P., Dell'Aversana, E., Carillo, P.: Spatial and temporal profile of glycinebetaine accumulation in plants under abiotic stresses. - Front. Plant Sci. 10: 230, 2019.

Banu, M.N.A., Hoque, M.A., Watanabe-Sugimoto, M., Islam, M.M., Uraji, M., Matsuoka, K., Nakamura, Y., Murata, Y.: Proline and glycinebetaine ameliorated $\mathrm{NaCl}$ stress via scavenging of hydrogen peroxide and methylglyoxal but not superoxide or nitric oxide in tobacco cultured cells. - Biosci. Biotechnol. Biochem. 74: 2043-2049, 2010. 
Baker, J.C., Mock, N.M.: An improved method for monitoring cell death in cell suspension and leaf disc assays using evans blue. - Plant Cell Tissue Organ Cult. 39: 7-12, 1994.

Bates, L.S., Waldren, R.P., Teare, I.D.: Rapid determination of free proline for water-stress studies. - Plant Soil 39: 205-207, 1973.

Berthold, D.A., Andersson, M.E., Nordlund, P.: New insight into the structure and function of the alternative oxidase. Biophys. Acta 1460: 241-254, 2000.

Bradford, M.M.: A rapid and sensitive method for the quantitation of microgram quantities of protein utilizing the principle of protein-dye binding. - Anal. Biochem. 72: 248-254, 1976.

Caliskan, O., Radusiene, J., Temizel, K.E., Staunis, Z., Cirak, C., Kurt, D., Odabas, M.S.: The effects of salt and drought stress on phenolic accumulation in greenhouse-grown Hypericum pruinatum. - Ital. J. Agron. 12: 271-275, 2017.

Cernoch, V., Kopecky, D.: Drought tolerance and regrowth capacity revealed in the Festuca-Lolium complex. - Biol. Plant. 64: 561-568, 2020

Chen, T.H.H., Murata, N.: Glycinebetaine: an effective protectant against abiotic stress in plants. - Trends Plant Sci. 13: 499$505,2008$.

Chen, W.P., Li, P.H., Chen, T.H.H.: Glycinebetaine increases chilling tolerance and reduces chilling-induced lipid peroxidation in Zea mays L. - Plant Cell Environ. 23: 609618, 2000.

Cisse, E.H.M., Miao, L.F., Yang, F., Huang, J.F., Li, D.D., Zhang, J.: Glycibetaine surpasses melatonin to improve salt tolerance in Dalbergia odorifera. - Front. Plant Sci. 12: 588847, 2021a.

Cisse, E.H.M., Zhang, L.J., Pu, Y.J., Miao, L.F., Li, DD., Zhang, J., Yang, F.: Exogenous $\mathrm{Ca}^{2+}$ associated with melatonin alleviates drought-induced damage in the woody tree Dalbergia odorifera. - J. Plant Growth Regul. 64, 2021 b.

Das, K., Roychoudhury, A.: Reactive oxygen species (ROS) and response of antioxidants as ROS-scavengers during environmental stress in plants. - Front. environ. Sci. 2: 53, 2014.

Demidchik, V.: Mechanisms of oxidative stress in plants: From classical chemistry to cell biology. - Environ. exp. Bot. 109: 212-228, 2015.

Demiral, T., Türkan, I.: Does exogenous glycinebetaine affect antioxidative system of rice seedlings under $\mathrm{NaCl}$ treatment? - J. Plant Physiol. 161: 1089-1100, 2004.

Ellman, G.L.: Tissue sulfhydryl groups. - Arch. Biochem. Biophys. 82: 70-77, 1959.

Escalante-Magaña, C., Aguilar-Caamal, L.F., EchevarríaMachado, I., Medina-Lara, F., Cach, L.S., Martínez-Estévez, M.: Contribution of glycinebetaine and proline to water deficit tolerance in pepper plants. - HortScience 54: 1044-1054, 2019.

Fan, J., Hu, Z., Xie, Y., Chan, Z., Chen, K., Amombo, E., Chen, L., Fu, J.: Alleviation of cold damage to photosystem II and metabolisms by melatonin in Bermudagrass. - Front. Plant Sci. 6: 925, 2015.

Farooq, M., Basra, S.M.A., Wahid, A., Cheema, Z.A., Cheema, M.A., Khaliq, A.: Physiological role of exogenously applied glycinebetaine to improve drought tolerance in fine grain aromatic rice (Oryza sativa L.). - J. Agron. Crop Sci. 194: 325-333, 2008.

Fielding, J.L., Hall, J.L.: A biochemical and cytochemical study of peroxidase activity in roots of Pisum sativum. - J. exp. Bot. 29: 983-991, 1978.

Khan, M.I.R., Asgher, M., Khan, N.A.: Alleviation of saltinduced photosynthesis and growth inhibition by salicylic acid involves glycinebetaine and ethylene in mungbean (Vigna radiata L.). - Plant Physiol. Biochem. 80: 67-74, 2014.
Kumar, P.: Stress amelioration response of glycine betaine and arbuscular mycorrhizal fungi in sorghum under Cr toxicity. PLoS ONE 16: e0253878, 2021a.

Kumar, P.: Soil applied glycine betaine with arbuscular mycorrhizal fungi reduces chromium uptake and ameliorates chromium toxicity by suppressing the oxidative stress in three genetically different sorghum (Sorghum bicolor L.) cultivars. - BMC Plant Biol. 21: 336, 2021 b.

Kumar, P.: Evaluation of toxic effects of hexavalent chromium on the yield and quality of sorghum. - Enliven J. Diet. Res. Nutr. 7: 1-11, 2020.

Kumar, P., Tokas, J., Singal, H.R.: Amelioration of chromium VI toxicity in sorghum (Sorghum bicolor L.) using glycine betaine. - Sci. Rep. 9: 1-15, 2019.

Li, X.W., Chen, Q.X., Lei, H.Q., Wang, J.W., Yang, S., Wei, H.X.: Nutrient uptake and utilization by fragrant rosewood (Dalbergia odorifera) seedlings cultured with oligosaccharide addition under different lighting spectra. - Forests 9: 29, 2018

Lichtenthaler, H.K., Wellburn, A.R.: Determinations of total carotenoids and chlorophylls $a$ and $b$ of leaf extracts in different solvents. - Biochem. Soc. Trans. 11: 591-592, 1983.

Ma, Q.Q., Wang, W., Li, Y.H., Li, D.Q., Zou, Q.: Alleviation of photoinhibition in drought-stressed wheat (Triticum aestivum) by foliar-applied glycinebetaine. - J. Plant Physiol. 163: 165175,2006

Ma, X.L., Wang, Y.J., Xie, S.L., Wang, C., Wang, W.: Glycine betaine application ameliorates negative effects of drought stress in tobacco. - Russ. J. Plant Physiol. 54: 472, 2007.

Mäkelä, P., Kleemola, J., Jokinen, K., Mantila, J., Pehu, E., Peltonen-Sainio, P.: Growth response of pea and summer turnip rape to foliar application of glycinebetaine. - Acta agr scand. Sect. B - Soil Plant Sci. 47: 168-175, 1997.

Mäkelä, P., Kontturi, M., Pehu, E., Somersalo, S.: Photosynthetic response of drought- and salt-stressed tomato and turnip rape plants to foliar-applied glycinebetaine. - Physiol. Plant. 105: 45-50, 1999.

Mäkelä, P., Mantila, J., Hinkkanen, R., Pehu, E., Peltonen-Sainio, P.: Effect of foliar applications of glycine betaine on stress tolerance, growth, and yield of spring cereals and summer turnip rape in Finland. - J. Agron. Crop Sci. 176: 223-234, 1996.

Malekzadeh, P.: Influence of exogenous application of glycinebetaine on antioxidative system and growth of saltstressed soybean seedlings (Glycine max L.). - Physiol. mol. Biol. Plants 21: 225-32, 2015.

Mróz, T.L., Havey, M.J., Bartoszewski, G.: Cucumber possesses a single terminal alternative oxidase gene that is upregulated by cold stress and in the mosaic (MSC) mitochondrial mutants. - Plant mol. Biol. Rep. 33: 1893-1906, 2015.

Osman, H.S.: Enhancing antioxidant-yield relationship of pea plant under drought at different growth stages by exogenously applied glycine betaine and proline. - Ann. agr. Sci. 60: 389402, 2015

Park, E.J., Jeknic, Z., Chen, T.H.H.: Exogenous application of glycine betaine increases chilling tolerance in tomato plants. Plant Cell Physiol. 47: 706-714, 2006.

$\mathrm{Pu}$, Y.-J., Cisse, E.H.M., Zhang, L.-J., Nawaz, M., Yang, F.: Coupling exogenous melatonin with $\mathrm{Ca}^{2+}$ alleviated chillimng stress in Dalbergia odorifera T. Chen. - Trees 65: 1541-1554, 2021.

Raza, M.A., Saleem, M., Shah, G., Khan, I., Raza, A.: Exogenous application of glycinebetaine and potassium for improving water relations and grain yield of wheat under drought. - J. Soil Sci. Plant Nutr. 14: 348-364, 2014.

Raza, S.H., Athar, H.R., Ashraf, M., Hameed, A.: Glycinebetaineinduced modulation of antioxidant enzymes activities and ion 
accumulation in two wheat cultivars differing in salt tolerance. - Environ. exp. Bot. 60: 368-376, 2007.

Ritonga, F.N., Chen, S.: Physiological and molecular mechanism involved in cold stress tolerance in plants. - Plants 9: 560, 2020.

Saha, B., Borovskii, G., Panda, S.K.: Alternative oxidase and plant stress tolerance. - Plant Signal. Behav. 11: e125630, 2016.

Shan, T., Jin, P., Zhang, Y., Huang, Y., Wang, X., Zheng, Y.: Exogenous glycine betaine treatment enhances chilling tolerance of peach fruit during cold storage. - Postharvest Biol. Technol. 114: 104-110, 2016.

Sharma, A., Kumar, V., Shahzad, B., Ramakrishnan, M., Singh Sidhu, G.P., Bali, A., Handa, N., Kapoor, D., Yadav, P., Khanna, K., Bakshi, P., Rehman, A., Kohli, S.K., Khan, E.A., Parihar, R.D., Yuan, H., Thukral, A.K., Bhardwaj, R., Zheng, B.: Photosynthetic response of plants under different abiotic stresses: a review. - J. Plant Growth Regul. 39: 509-531, 2020.

Sharma, A., Shahzad, B., Kumar, V., Kohli, S.K., Sidhu, G.P.S., Bali, A.S., Handa, N., Kapoor, D., Bhardwaj, R., Zheng, B.: Phytohormones regulate accumulation of osmolytes under abiotic stress. - Biomolecules 9: 285, 2019.

Sies, H.: Strategies of antioxidant defence. - Eur. J. Biochem. 215: 213-219, 1993.

Staniak, M., Bojarszczuk, J., Kraska, P., Kwiatkowski, C., Harasim, E.: Prolonged drought stress induced changes in yield and physiological processes of Trifolium repens and Festulolium braunii. - Biol. Plant. 64: 701-709, 2020.

Swain, T., Hillis, W.E.: The phenolic constituents of Prunus domestica. I. The quantitative analysis of phenolic constituents. - J. Sci. Food Agr. 10: 63-68, 1959.
Tian, F., Wang, W., Liang, C., Wang, X., Wang, G., Wang, W.: Overaccumulation of glycine betaine makes the function of the thylakoid membrane better in wheat under salt stress. Crop J. 5: 73-82, 2017.

Varela, M.C., Arslan, I., Reginato, M.A., Cenzano, A.M., Luna, M.V.: Phenolic compounds as indicators of drought resistance in shrubs from Patagonian shrublands (Argentina). - Plant Physiol. Biochem. 104: 81-91, 2016.

Wanner, L.A., Junttila, O.: Cold-induced freezing tolerance in Arabidopsis. - Plant Physiol. 120: 391-400, 1999.

Wikberg, J., Ogren, E.: Variation in drought resistance, drought acclimation and water conservation in four willow cultivars used for biomass production. - Tree Physiol. 27: 1339-1346, 2007.

Xu, Z., Sun, M., Jiang, X., Sun, H., Dang, X., Cong, H., Qiao, F.: Glycine betaine biosynthesis in response to osmotic stress depends on jasmonate signaling in watermelon suspension cells. Front. Plant Sci. 9: 1469, 2018.

Yancey, P.H.: Compatible and counteracting solutes. - In Strange, K. (ed.): Cellular and Molecular Physiology of Cell Volume Regulation. Vol. 5. Pp. 81-109. CRC-Press, Boca Raton 1994.

Yang, F., Wang, Y., Miao, L.F.: Comparative physiological and proteomic responses to drought stress in two poplar species originating from different altitudes. - Physiol. Plant. 139: 388400, 2010.

Yemm, E.W., Willis, A.J.: The estimation of carbohydrates in plant extracts by anthrone. - Biochem. J. 57: 508-514, 1954.

Zagorchev, L., Seal, C., Kranner, I., Odjakova, M.: A central role for thiols in plant tolerance to abiotic stress. - Int. J. Mol. Sci. 14: 7405-32, 2013. 